\title{
Dosimetric implications of two registration based patient positioning methods in prostate image guided radiation therapy (IGRT)
}

\author{
D Ryan C Rivest ${ }^{1,3}$, Terence A Riauka ${ }^{2,3}$, Albert D Murtha ${ }^{2,4}$, B Gino Fallone ${ }^{1,2,3}$ \\ ${ }^{1}$ Department of Physics, ${ }^{2}$ Department of Oncology, University of Alberta, \\ ${ }^{3}$ Department of Medical Physics, ${ }^{4}$ Department of Radiation Oncology, \\ Cross Cancer Institute, Edmonton, Alberta, Canada
}

\begin{abstract}
Background. We compare the dosimetry of daily patient positioning based on prostate matching versus bone matching for patients treated with helical tomotherapy.

Methods. Ninety-nine pre-treatment 3D megavoltage (MV) CT images of four high risk prostate patients were registered to their respective planning images using two automatic registration algorithms, one achieving bone matching and the other prostate matching. Dose distributions that would have been delivered had patient positioning been based on each matching method were evaluated. Contours were delineated on each MVCT image and prostate, bladder, and rectum dose volume histograms were compared for each image guidance strategy using endpoints adapted from inverse planning constraints.

Results. The standard deviation of per fraction prostate $\triangle D 95$ values, defined as prostate matching D95 minus bone matching D95, was 0.01 Gy (Range: -0.02 to 0.02 Gy). Defined analogously, bladder $\Delta D 45$ and rectum $\triangle D 30$ values were $0.12 \mathrm{~Gy}$ (Range: -0.22 to $0.52 \mathrm{~Gy}$ ) and $0.14 \mathrm{~Gy}$ (Range: -0.40 to $0.34 \mathrm{~Gy}$ ), respectively.

Conclusions. Bladder $\triangle D 45$ and rectum $\triangle D 30$ standard deviation values corresponding to $6.1 \%$ and $7.5 \%$ of their respective planning constraints suggesting critical structure doses are dependent on positioning method. A relationship between critical structure dosimetry and the direction of daily prostate motion was also observed.
\end{abstract}

Key words: prostate radiotherapy; helical tomotherapy; patient positioning; image registration

Received 4 June 2009

Accepted 7 July 2009

Correspondence to: B Gino Fallone, PhD, Department of Medical Physics, Cross Cancer Institute, 11560 University Avenue, Edmonton, Alberta, Canada T6G 1Z2. Phone: 1780432 8750; Fax: 1780432 8615; E-mail: gfallone@phys.ualberta.ca

\section{Background}

The field of image-guided radiation therapy (IGRT) rose from the need to account for daily anatomical variations in the delivery of fractionated radiation therapy. This is particularly relevant to the treatment of prostate cancer as it has been repeatedly 


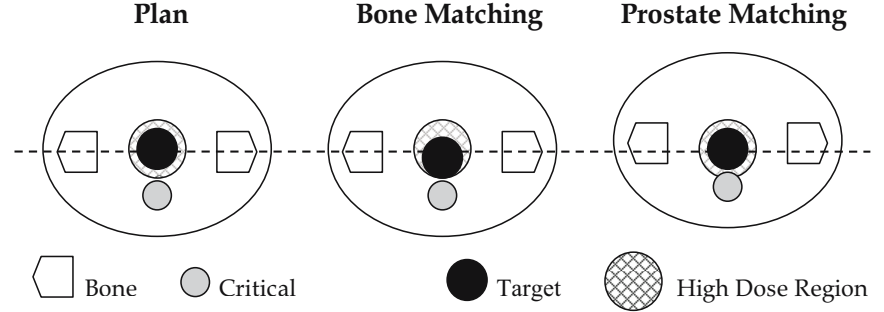

Figure 1. Simplified schematic demonstrating the potential increased dose to a critical structure as a result of prostate (i.e. target) matching. A gap is present between the target and critical structure in the planning $\mathrm{CT}$, but the target has moved towards the critical structure when the patient is treated. With appropriate margins, bone matching results in complete coverage of the target without having any of the critical structure situated in the high dose region. On the other hand, part of the critical structure receives the target dose if prostate matching is used for patient positioning.

demonstrated that the position of the prostate gland varies as a result of bladder and rectal filling. ${ }^{1,2}$ A number of correctional strategies including implanted fiducials ${ }^{3-5}$ and on-line three-dimensional (3D) computed tomography (CT) imaging ${ }^{6-9}$ have been developed and clinically implemented. Although both methods provide daily image guidance, the latter is advantageous in that it allows for the evaluation of daily delivered doses. ${ }^{10}$

The principle modality for prostate IGRT at our clinic is the $\mathrm{Hi}^{*}$ Art II helical tomotherapy unit (TomoTherapy, Inc. Madison, WI). Prior to each fraction, clinicians acquire a 3D megavoltage CT (MVCT) of the patient in treatment position which is subsequently registered to the patient's planning $\mathrm{CT}$ and based on the registration; the patient is repositioned and treated. To ensure the target is situated in the same geometric location on a daily basis, registration should result in the overlap of the prostate volumes in the MVCT and planning CT images. This varies from traditional prostate treatment protocols where the lack of soft tissue contrast in portal images made prostate matching impossible and patient re-positioning was based on the matching of bony anatomy. ${ }^{11-16}$ This change in patient positioning method- ology has potential detrimental consequences to the dosimetry of critical structures. For example, consider the scenario where the prostate has moved slightly in the direction of a critical structure. Bone matching, in combination with sufficient margins, will ensure the entire target receives the prescription dose, however, prostate matching would certainly lead to an increased dose to the critical structure (Figure 1). Unfortunately, the dosimetric implications of using prostate matching instead of bone matching for daily image guidance have not been sufficiently investigated. The objective of this work is to quantify and compare the doses that would be delivered to the prostate, bladder, and rectum if image guidance on the $\mathrm{Hi}^{*}$ Art II system was based on prostate matching or on bone matching. The dependence of dosimetric variations on the direction of daily prostate motion will also be investigated.

\section{Methods}

Treatment data from four research patients treated for high risk prostate cancer on the $\mathrm{Hi}^{*}$ Art II unit were available for this local research ethics board approved retrospective study. The primary planning target volume (PTV), treated to 68.0 Gy over 25 fractions (2.72 Gy/fraction), was defined by margins of $7-\mathrm{mm}$ posteriorly and $10-\mathrm{mm}$ in all other directions around the prostate gland and seminal vesicles. Constraints for the rectum and bladder during inverse planning were that no more than $30 \%$ of the rectum volume receive 45.0 Gy (1.80 Gy/fraction) and no more than $45 \%$ of the bladder volume receive 50.0 Gy (2.00 
Gy/per fraction). Patients were instructed to have a full bladder and empty rectum during simulation and each daily treatment fraction. Prior to each fraction, a pelvic MVCT was acquired and used for patient re-positioning. For our purposes, one MVCT was removed from the data set because the entire prostate was not imaged, leaving ninety-nine fractions available for this study.

As a result of daily prostate motion with respect to rigid pelvic bony anatomy, prostate matching and bone matching of daily MVCT and planning images produces two different image alignments. ${ }^{17}$ In this work, prostate matching and bone matching was performed using in-house developed automatic rigid registration software. For prostate matching, daily MVCT images were registered to planning $\mathrm{CT}$ images by optimizing the correlation coefficient metric. To ensure the overlap of the MVCT and planning CT prostate volumes, only the planning CT voxels corresponding to prostate plus a small $6.0-\mathrm{mm}$ border were used in cost function calculation. ${ }^{18-20}$ Voxels in the border region corresponding to bone and intestinal gas were filtered via thresholding to eliminate their influence on registration. In addition, a noise reducing median filter $^{21}$ was applied to MVCT images prior to registration. For bone matching, we used the mutual information algorithm proposed by Mattes et al. ${ }^{22}$, however, only the automatically segmented planning CT voxels corresponding to bony anatomy were used to evaluate the cost function. Both the prostate and bone matching procedures rely on the Nelder-Mead simplex algorithm for cost function optimization. ${ }^{23}$

Following completion of bone matching and prostate matching procedures, dose distributions that would have been delivered using both image guidance strategies were evaluated with the High*Art II system's inherent Planned Adaptive software.
The software evaluates delivered dose distributions by applying the treatment delivery sinogram to daily MVCT images, and has been demonstrated to have dosimetric accuracy comparable to that of planning CT dose calculations. ${ }^{10} \mathrm{~A}$ number of studies have been published in which the tool was used to compare planned and delivered doses 24,25 , however, to the best of our knowledge, the software has not been used to calculate theoretical dose distributions that would have been delivered had patient positioning been performed differently.

Retrospectively, prostate, rectum, and bladder volumes were contoured by a radiation oncologist on all pre-treatment MVCT images using the Planned Adaptive software and dose volume histograms (DVH) were evaluated for each dose distribution. Structures on each MVCT image were delineated only once and external software was used to account for the translational shifts in the contour co-ordinates between the two matching methods. Dosimetric endpoints for DVH analysis were adopted from the tumour and sensitive structure constraints implemented during inverse planning. As such, values for D95, D45, and D30 were extracted from each prostate, bladder, and rectum DVH, respectively. In addition to absolute evaluation, the differences between endpoints for each method were also evaluated. ${ }^{26}$ As such, for comparison of prostate matching and bone matching image guidance strategies, $\triangle \mathrm{D} 95$, defined as prostate D95 for prostate matching minus prostate D95 for bone matching, was determined for each fraction. Defined analogously, bladder $\Delta \mathrm{D} 45$ and rectum $\Delta \mathrm{D} 30$ values were also evaluated. In addition, five MVCT images were selected at random and re-contoured upon which dosimetric analysis was repeated to determine the effect of uncertainties in structure delineation on the evaluated endpoints. 


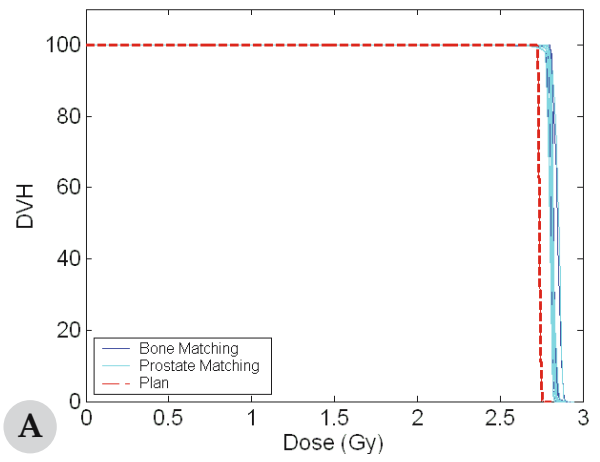

Figure 2. DVH comparison of prostate matching (PM) and bone matching (BM) for patient 1 odd numbered fractions (i.e. fraction 1, 3, ... 23, 25). (a) Prostate, (b) bladder, (c) rectum.

The relationship between bladder ${ }^{27,28}$ and rectal $^{27-29}$ filling and prostate motion in the superior/inferior (SI) and anterior/posterior (AP) directions has been repeatedly documented in the literature. Bladder D45 and rectum D30 values are clearly dependent on organ volumes, and as such, endpoints were also evaluated with respect to the measured daily prostate motion. Daily prostate motion (i.e. for each fraction) was calculated from the differences between the bone matching and prostate matching alignments.

\section{Results}

\section{Daily prostate motion}

The mean ( \pm standard deviation) of the prostate motion for the ninety-nine fraction cohort was $2.4 \pm 1.9 \mathrm{~mm}$ superiorly, 0.7 $\pm 2.5 \mathrm{~mm}$ anteriorly, and $0.3 \pm 0.5 \mathrm{~mm}$ to the left. Although motion was skewed superiorly, standard deviations are comparable with values previously reported in the literature, ${ }^{1}$ albeit on the lower end. Statistics for each individual patient are reported in Table 1.

\section{Dose volume histogram analysis}

The mean ( \pm standard deviation) prostate D95, bladder D45 and rectum D30 val-
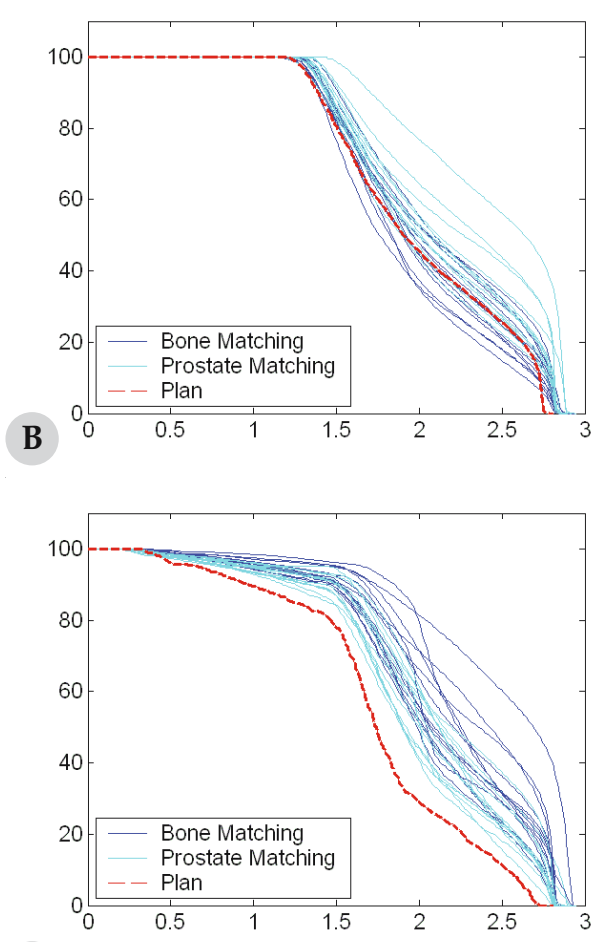

C

ues for each matching method are given in Table 2. Although mean prostate D95 values are identical for prostate matching and bone matching, evaluated critical structure endpoints are dependent on the matching method. Worth mentioning is the fact that all reported values exceed the aforementioned inverse planning constraints. In light of these observations, the percentage of fractionated bladder D45 and rectum D30 values that exceeded the constraints by differing dosimetric amounts were evaluated and appear in Table 3. Observed bladder and rectum endpoints correlate with the observed trends of anterior and superior prostate motion reported in the previous section. Presumably, if the prostate has moved superiorly towards the bladder, prostate matching would increase the volume of bladder that receives the prescription dose. Similarly, anterior prostate motion away from the rectum would result 
Table 1. Prostate motion statistics for each individual patient. Negative values correspond to motion superiorly, anteriorly and to the left

\begin{tabular}{llll}
\hline Patient & Direction & Mean \pm SD $(\mathbf{m m})$ & Range $(\mathbf{m m})$ \\
\hline \multirow{2}{*}{2} & LR & $0.0 \pm 0.4$ & -0.8 to 0.8 \\
\cline { 2 - 4 } & AP & $-2.3 \pm 3.2$ & -8.3 to 8.4 \\
\cline { 2 - 4 } & SI & $-4.1 \pm 1.6$ & -8.0 to -1.6 \\
\hline \multirow{2}{*}{3} & LR & $-0.1 \pm 0.4$ & -1.0 to 0.7 \\
\cline { 2 - 4 } & AP & $0.0 \pm 1.5$ & -3.3 to 3.0 \\
\cline { 2 - 4 } & SI & $-2.1 \pm 1.2$ & -6.7 to -0.2 \\
\hline \multirow{4}{*}{4} & LR & $-0.8 \pm 0.4$ & -1.7 to -0.3 \\
\cline { 2 - 4 } & AP & $-1.7 \pm 1.9$ & -5.3 to 1.9 \\
\cline { 2 - 4 } & SI & $-1.1 \pm 2.0$ & -4.7 to 2.8 \\
\cline { 2 - 4 } & LR & $-0.3 \pm 0.6$ & -2.1 to 0.4 \\
\hline \multirow{nyyy}{*}{ Combined } & AP & $-2.9 \pm 1.8$ & -2.3 to 6.5 \\
\cline { 2 - 4 } & LR & $-0.3 \pm 0.5$ & -2.0 to 2.4 \\
\cline { 2 - 4 } & AP & $-0.7 \pm 2.5$ & -8.3 to 8.4 \\
\cline { 2 - 4 } & SI & $-2.4 \pm 1.9$ & -8.0 to 2.8 \\
\hline
\end{tabular}

$\triangle \mathrm{D} 45$ values ranged from -0.22 to $0.52 \mathrm{~Gy}$, having a mean value of $0.07 \pm 0.12$ Gy. Finally, the mean rectum $\Delta \mathrm{D} 30$ value was $-0.06 \pm 0.14$ $\mathrm{Gy}$, with values ranging from -0.40 to 0.34 Gy. Statistics for each individual patient are reported in Table 4. Measured bladder $\triangle \mathrm{D} 45$ and rectum $\Delta \mathrm{D} 30$ values are plotted as a function of prostate motion in the $\mathrm{AP}$ and SI directions in Figure 4. A dependence on the direction of prostate motion is clearly evident.

\section{Contour dependence}

The absolute difference between endpoints evaluated using original and repeat contours were calculated for each of the five re-contoured MVCT images. The mean

in the prescription dose being delivered to less rectal volume. The thirteen odd numbered fraction dose volume histograms for patient 1 are plotted in Figure 2 to demonstrate the daily variations in prostate, bladder and rectum dosimetry for each matching method.

\section{Image guidance comparison}

Histograms of the theoretical prostate $\Delta \mathrm{D} 95$, bladder $\Delta \mathrm{D} 45$, and rectum $\Delta \mathrm{D} 30$ values for all ninety-nine fractions are displayed in Figure 3. The mean ( \pm standard deviation) prostate $\Delta \mathrm{D} 95$ for the cohort was $0.00 \pm 0.01 \mathrm{~Gy}$, with values ranging from -0.02 to 0.02 Gy. Observed bladder

Table 2. Per fraction mean \pm standard deviation prostate D95, bladder D45 and rectum D30 values for all ninety-nine fractions for each matching method. Inverse planning constraints are given in brackets

\begin{tabular}{lcc}
\hline & Bone Matching (Gy) & Prostate Matching (Gy) \\
\hline Prostate D95 ( $\geq 2.72 \mathrm{~Gy})$ & $2.81 \pm 0.02$ & $2.81 \pm 0.02$ \\
Bladder D45 $(\leq 2.00 \mathrm{~Gy})$ & $2.06 \pm 0.17$ & $2.13 \pm 0.21$ \\
Rectum D30 $(\leq 1.80 \mathrm{~Gy})$ & $2.29 \pm 0.22$ & $2.23 \pm 0.17$ \\
\hline
\end{tabular}

( \pm standard deviation) absolute difference of the ten (five for each matching method) bladder D45 values was $0.03 \pm 0.02 \mathrm{~Gy}$. This represents $1.2 \pm 0.7 \%$ of the prescription dose. Corresponding values for rectum D30 absolute differences were slightly higher at $0.07 \pm 0.07 \mathrm{~Gy}$ or, $2.5 \pm 2.6 \%$ of the prescription dose. The mean $( \pm$ standard deviation) of the five bladder $\Delta \mathrm{D} 45$ absolute differences was $0.01 \pm 0.02$ Gy or $0.3 \pm 0.6 \%$ of the prescription dose. Rectum $\Delta \mathrm{D} 30$ values were $0.04 \pm 0.04$ Gy or $1.4 \pm$ $1.2 \%$. Mean and standard deviations of the absolute differences in both prostate D95 and prostate $\Delta$ D95 values were less than $0.1 \%$ of the prescription dose. Based on these observations, the reported magnitudes of prostate $\triangle \mathrm{D} 95$, bladder $\triangle \mathrm{D} 45$ and rectum $\triangle \mathrm{D} 30$ values exceed their uncertainties relating to errors in contour delineation. 


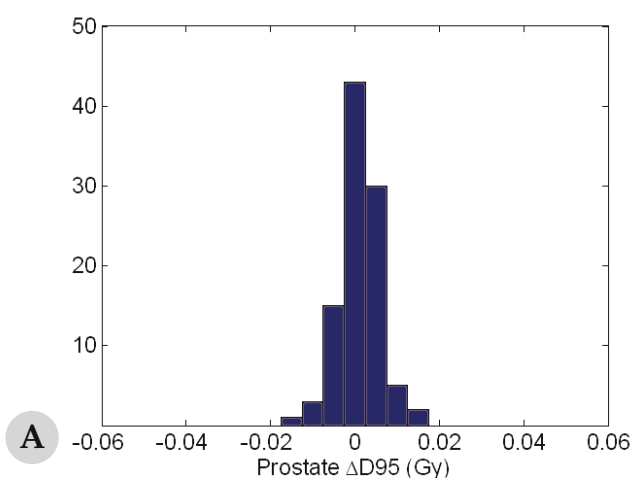

Figure 3. Frequency histograms of the theoretical fractionated (a) prostate $\Delta \mathrm{D} 95$, (b) bladder $\Delta \mathrm{D} 45$, and (c) rectum $\Delta \mathrm{D} 30$ values for all ninety-nine fractions.

\section{Discussion}

The image guidance protocol mandated at our clinic during treatment of the high risk prostate patients on helical tomotherapy, including those analyzed in this study, is as follows. After initial automatic registration of daily MVCT and planning CT images, the alignment is manually adjusted by a radiation therapist with the goal of overlapping the prostate gland in the two images using the prostate/rectum interface at the mid-plane of the prostate as a reference. Recently, Langen et al. ${ }^{30}$ investigated the accuracy of a manual method similar to ours and found that over 224 manual registrations performed by two radiation therapists, prostate misalignment exceeded $3-\mathrm{mm} 24 \%, 33 \%$, and $3 \%$ of the time in the $\mathrm{AP}, \mathrm{SI}$, and LR directions, respectively.

\section{B}

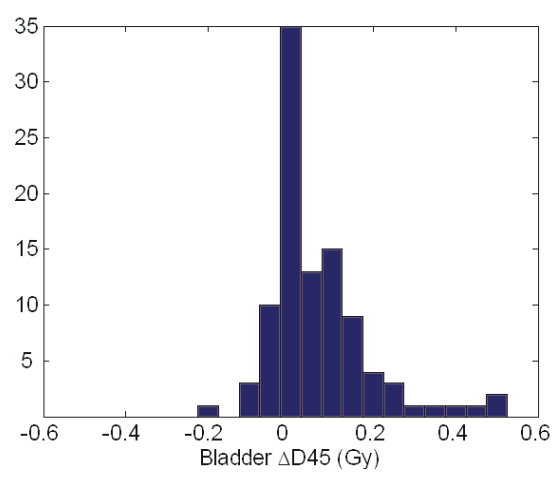

C

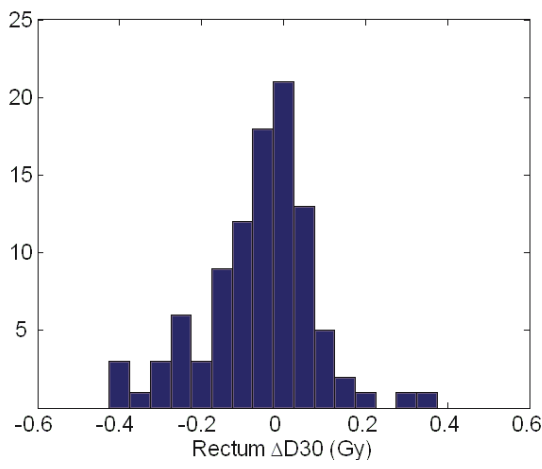

Misalignment never exceeded 5-mm in any single direction. Although our work compares image matching strategies specifically, the observed alignment differences between prostate and bone matching are representative of the range of possible prostate misalignments during treatment. As such, the prostate $\triangle \mathrm{D} 95$ values observed in this study suggest that for the margins used clinically and the errors associated with the manual patient positioning method employed at our centre, delivered prostate D95 values are unaffected by observed prostate positioning errors. This suggests that daily image guidance based on prostate matching of treatment and planning CT images allows for a reduction of the 10 mm margins used for 
Table 4. Individual patient statistics for prostate $\Delta \mathrm{D} 95$, bladder $\Delta \mathrm{D} 45$ and rectum $\Delta$ D30. $\Delta$ denotes prostate matching minus bone matching. The combined patient values are also normalized to the inverse planning constraints (i.e. prostate: $2.72 \mathrm{~Gy}$, bladder: $2.00 \mathrm{~Gy}$ and rectum: $1.80 \mathrm{~Gy}$ )

\begin{tabular}{llll}
\hline Patient & & $\begin{array}{l}\text { Standard } \\
\text { Deviation (Gy) }\end{array}$ & Range (Gy) \\
\hline \multirow{2}{*}{3} & Prostate $\Delta \mathrm{D} 95$ & $<0.01$ & -0.01 to 0.00 \\
\cline { 2 - 4 } & Bladder $\Delta \mathrm{D} 45$ & 0.16 & -0.22 to 0.52 \\
\cline { 2 - 4 } & Rectum $\Delta \mathrm{D} 30$ & 0.15 & -0.40 to 0.32 \\
\hline \multirow{2}{*}{3} & Prostate $\Delta \mathrm{D} 95$ & $<0.01$ & -0.02 to 0.01 \\
\cline { 2 - 4 } & Bladder $\Delta \mathrm{D} 45$ & 0.05 & -0.09 to 0.17 \\
\cline { 2 - 4 } & Rectum $\Delta \mathrm{D} 30$ & 0.09 & -0.26 to 0.22 \\
\hline \multirow{2}{*}{4} & Prostate $\Delta \mathrm{D} 95$ & $<0.01$ & 0.00 to 0.01 \\
\cline { 2 - 4 } & Bladder $\Delta \mathrm{D} 45$ & 0.08 & -0.08 to 0.20 \\
\cline { 2 - 4 } & Rectum $\Delta \mathrm{D} 30$ & 0.10 & -0.34 to 0.06 \\
\cline { 2 - 4 } & Prostate $\Delta \mathrm{D} 95$ & $<0.01$ & 0.00 to 0.02 \\
\cline { 2 - 4 } & Bladder $\Delta \mathrm{D} 45$ & 0.04 & -0.08 to 0.08 \\
\hline \multirow{2}{*}{ Combined } & Pectum $\Delta \mathrm{D} 30$ & 0.09 & -0.10 to 0.34 \\
\cline { 2 - 4 } & Plostate $\Delta \mathrm{D} 95$ & $<0.01(0.2 \%)$ & -0.02 to $0.02(-0.6$ to $0.6 \%)$ \\
\cline { 2 - 4 } & Rectum $\Delta \mathrm{D} 30$ & $0.14(7.5 \%)$ & -0.40 to $0.34(-22.0$ to $19.1 \%)$ \\
\hline
\end{tabular}

the patients included in this study. Recent work by Meijer et al. ${ }^{31}$ demonstrated that a prostate margin of $6 \mathrm{~mm}$ is clinically acceptable when daily image guidance is based on the alignment of implanted fiducials. Without fiducials, as is the case for the helical tomotherapy patients at our centre, daily prostate positioning errors will increase ${ }^{30}$ meaning a $6 \mathrm{~mm}$ margin is insufficient. Further investigation is required to determining where in the 6 to $10 \mathrm{~mm}$

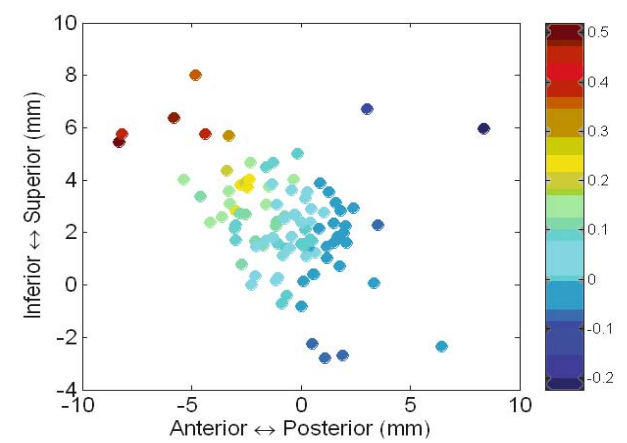

range that the acceptable margin for prostate matching without fiducials is situated.

In relation to the two critical structures, the observed ranges of bladder $\triangle \mathrm{D} 45$ and rectum $\triangle \mathrm{D} 30$ values in combination with the fact that reported mean bladder D45 and rectum D30 values exceed inverse planning constraints, suggests that the selection of matching procedure has clinically significant repercussions on the dosimetry of the critical structures. However, over the entire ninety-nine fraction cohort analyzed in this study, positive and negative bladder $\triangle \mathrm{D} 45$ and rectum $\Delta \mathrm{D} 30$ values offset each other, giving rise to mean values of $2.6 \%$ and $-2.2 \%$ of the prescription dose, respectively. How this fractionated trend translates when comparing matching methods based on the cumulative critical structure dosimetry over entire treatments remains unanswered.

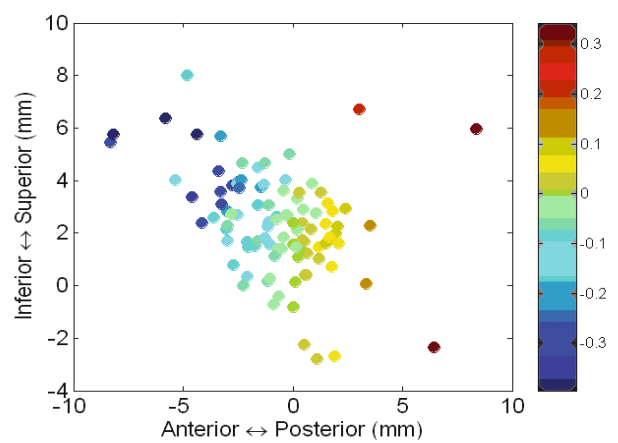

Figure 4. Scatter plot of per fraction (a) bladder $\Delta \mathrm{D} 45$ and (b) rectum $\Delta \mathrm{D} 30$ values for all ninety-nine fractions as a function of prostate motion in the superior/inferior and anterior/posterior directions. 
Investigating this requires accurate deformable registration to track daily anatomical variations ${ }^{32,33}$ and will be the focus of future work. However, whether or not fractionated dosimetrical differences between matching methods cancel out over prolonged treatment regiments, the fractionated analysis reported in this paper is beneficial for a number of reasons. First, the easiest way to ensure treatment protocols are satisfied over protracted treatment regiments is to ensure those same protocols are satisfied each fraction. Furthermore, the radiobiological effects of varying daily doses differ from those associated with static daily dosimetry, regardless of whether the cumulative dosimetry is equivalent. Finally, results demonstrate that systematic trends in daily prostate motion for individual patients can lead to large discrepancies in the dosimetry of prostate and bone matching. Patient 1 for example, had consistently and often significantly less bladder volume in daily treatment images as compared to planning CT acquisition, which contributed to a mean superior $4.1 \mathrm{~mm}$ prostate shift over all twenty-five fractions. ${ }^{28}$ This systematic prostate motion resulted in a mean bladder $\Delta \mathrm{D} 45$ of $0.20 \mathrm{~Gy}$, which represents $10.0 \%$ of the inverse planning D45 constraint, suggesting that the selection of daily matching strategies can potentially have a clinically significant effect on cumulative critical structure dosimetry as well.

\section{Conclusions}

We have used the Planned Adaptive software on the $\mathrm{Hi}^{*}$ Art II system to compare the doses that would have been delivered to high risk prostate patients if daily patient re-positioning was based on bone matching versus prostate matching. DVH analysis demonstrates that the difference in prostate dose for each matching technique is insignificant, allowing for potential margin reduction. However, observed ranges in the differences between critical structure dosimetry for bone and prostate matching suggest that the selection of matching method employed during patient re-positioning has clinical repercussions. In fact, the doses delivered to the bladder and the rectum were found to be highly dependent not only on the image guidance strategy, but also the direction of daily prostate motion. In particular, for prostate motion anteriorly and superiorly, bone matching decreases bladder dose whereas prostate matching decreased the rectal dose. Potentially, the matching method can be selected each day based on the observed prostate motion in order to minimize dose and subsequent complications to the bladder and rectum.

\section{References}

1. Langen KM, Jones DTL. Organ motion and its management. Int J Radiat Oncol Biol Phys 2001; 50: 265-78.

2. Byrne TE. A review of prostate motion with considerations for the treatment of prostate cancer. Med Dosim 2005; 30: 155-61.

3. Nederveen AJ, Dehnad $\mathrm{H}$, van der Heide UA, van Moorselaar RJ, Hofman P, Lagendijk JJ. Comparison of megavoltage position verification for prostate irradiation based on bony anatomy and implanted fiducials. Radiother Oncol 2003; 68: 81-8.

4. Willoughby TR, Kupelian PA, Pouliot J, Shinohara K, Aubin M, Roach M 3rd, et al. Target localization and real-time tracking using the Calypso 4D localization system in patients with localized prostate cancer. Int J Radiat Oncol Biol Phys 2006; 65: 528-34.

5. Chen J, Lee RJ, Handrahan D, Sause WT. Intensitymodulated radiotherapy using implanted fiducial markers with daily portal imaging: assessment of prostate organ motion. Int J Radiat Oncol Biol Phys 2007; 68: 912-9.

6. Cheng CW, Wong J, Grimm L, Chow M, Uematsu $\mathrm{M}$, Fung A. Commissioning and clinical implementation of a sliding gantry CT scanner installed in an existing treatment room and early clinical experience for precise tumor localization. Am J Clin Oncol 2003; 26: e28-36. 
7. Wong JR, Grimm L, Uematsu M, Oren R, Cheng $\mathrm{CW}$, Merrick $\mathrm{S}$, et al. Image-guided radiotherapy for prostate cancer by CT-linear accelerator combination: prostate movements and dosimetric considerations. Int J Radiat Oncol Biol Phys 2005; 61: $561-9$.

8. Sorcini B, Tilikidis A. Clinical application of image-guided radiotherapy, IGRT (on the Varian OBI platform). Cancer Radiother 2006; 10: 252-7.

9. Kupelian PA, Lee C, Langen KM, Zeidan OA, Ma on RR, Willoughby TR, et al. Evaluation of image-guidance strategies in the treatment of localized prostate cancer. Int J Radiat Oncol Biol Phys 2008; 70: 1151-7.

10. Langen KM, Meeks SL, Poole DO, Wagner TH, Willoughby TR, Kupelian PA, et al. The use of megavoltage CT (MVCT) images for dose recomputations. Phys Med Biol 2005; 50: 4259-76.

11. Cionini L, Bucciolini M. Role of portal imaging in clinical radiotherapy: Florence experience. Radiother Oncol 1993; 29: 230-6.

12. Gilhuijs KG, el-Gayed AA, van Herk M, Vijlbrief RE. An algorithm for automatic analysis of portal images: clinical evaluation for prostate treatments. Radiother Oncol 1993; 29: 261-8.

13. Althof VG, Hoekstra CJ, te Loo HJ. Variation in prostate position relative to adjacent bony anatomy. Int J Radiat Oncol Biol Phys 1996; 34: 709-15.

14. Bieri S, Miralbell R, Nouet P, Delorme H, Rouzaud M. Reproducibility of conformal radiation therapy in localized carcinoma of the prostate without rigid immobilization. Radiother Oncol 1996; 38: 223-30.

15. Mubata CD, Bidmead AM, Ellingham LM, Thompson V, Dearnaley DP. Portal imaging protocol for radical dose-escalated radiotherapy treatment of prostate cancer. Int J Radiat Oncol Biol Phys 1998; 40: 221-31.

16. Greer PB, Mortensen TM, Jose CC. Comparison of two methods for anterior-posterior isocenter localization in pelvic radiotherapy using electronic portal imaging. Int J Radiat Oncol Biol Phys 1998; 41: 1193-9.

17. Fiorino C, Di Muzio N, Broggi S, Cozzarini C, Maggiulli E, Alongi F, et al. Evidence of limited motion of the prostate by carefully emptying the rectum as assessed by daily MVCT image guidance with helical tomotherapy. Int J Radiat Oncol Biol Phys 2008; 71: 611-7.
18. Court LE, Dong L. Automatic registration of the prostate for computed-tomography-guided radiotherapy. Med Phys 2003; 30: 2750-7.

19. Smitsmans MHP, Wolthaus JWH, Artignan X, de Bois J, Jaffray DA, Lebesque JV, et al. Automatic localization of the prostate for on-line or off-line image-guided radiotherapy. Int J Radiat Oncol Biol Phys 2004; 60: 623-35.

20. Smitsmans MHP, De Bois J, Sonke JJ, Betgen A, Zijp LJ, Jaffray DA, et al. Automatic prostate localization on cone-beam CT scans for high precision image-guided radiotherapy. Int J Radiat Oncol Biol Phys 2005; 63: 975-84.

21. Lau YH, Braun M, Hutton BF. Non-rigid image registration using a median-filtered coarse-to-fine displacement field and a symmetric correlation ratio. Phys Med Biol 2001; 46: 1297-319.

22. Mattes D, Haynor DR, Vesselle H, Lewellen TK, Eubank W. PET-CT image registration in the chest using free-form deformations. IEEE Trans Med Imag 2003; 22: 120-8.

23. Nelder JA, Mead R. A simplex method for function minimization. Comput J 1965; 7: 308-13.

24. Kupelian PA, Langen KM, Zeidan OA, Meeks SL, Willoughby TR, Wagner TH, et al. Daily variations in delivered doses in patients treated with radiotherapy for localized prostate cancer. Int J Radiat Oncol Biol Phys 2006; 66: 876-82.

25. Han C, Chen YJ, Liu A, Schultheiss TE, Wong JY. Actual dose variation of parotid glands and spinal cord for nasopharyngeal cancer patients during radiotherapy. Int J Radiat Oncol Biol Phys 2008; 70: 1256-62.

26. Grabec D, Kragelj B. The sigmoid colon and bladder shielding in whole pelvic irradiation of prostate cancer (forward planned IMRT from Institute of Oncology Ljubljana). Radiol Oncol 2009; 43: 56-64.

27. Roeske JC, Forman JD, Mesina CF, He T, Pelizzari CA, Fontenla E, et al. Evaluation of changes in the size and location of the prostate, seminal vesicles, bladder, and rectum during a course of external beam radiation therapy. Int J Radiat Oncol Biol Phys 1995; 33: 1321-9.

28. Melian E, Mageras GS, Fuks Z, Leibel SA, Niehaus A, Lorant $\mathrm{H}$, et al. Variation in prostate position quantitation and implications for threedimensional conformal treatment planning. Int $J$ Radiat Oncol Biol Phys 1997; 38: 73-81. 
29. van Herk M, Bruce A, Kroes AP, Shouman T, Touw A, Lebesque JV. Quantification of organ motion during conformal radiotherapy of the prostate by three dimensional image registration. Int J Radiat Oncol Biol Phys 1995; 33: 1311-20.

30. Langen KM, Zhang Y, Andrews RD, Hurley ME, Meeks SL, Poole DO, et al. Initial experience with megavoltage (MV) CT guidance for daily prostate alignments. Int J Radiat Oncol Biol Phys 2005; 62: 1517-24.

31. Meijer GJ, de Klerk J, Bzdusek K, van den Berg HA, Janssen R, Kaus MR, et al. What CTV-to-PTV margins should be applied for prostate irradiation? Four-dimensional quantitative assessment using model-based deformable image registration techniques. Int J Radiat Oncol Biol Phys 2008; 72: 1416-25.

32. Schaly B, Kempe JA, Bauman GS, Battista JJ, Van Dyk J. Tracking the dose distribution in radiation therapy by accounting for variable anatomy. Phys Med Biol 2004; 49: 791-805.

33. Lu W, Olivera GH, Chen Q, Ruchala KJ, Haimerl J, Meeks SL, et al. Deformable registration of the planning image $(\mathrm{kVCT})$ and the daily images (MVCT) for adaptive radiation therapy. Phys Med Biol 2006; 51: 4357-74. 